\title{
L'Administration nationale de l'éducation publique en Uruguay : une institution unique au monde
}

Damien Larrouqué

\section{(2) OpenEdition}

\section{Journals}

Édition électronique

URL : https://journals.openedition.org/ries/6326

DOI : $10.4000 /$ ries. 6326

ISSN : 2261-4265

Éditeur

France Education international

Édition imprimée

Date de publication : 1 septembre 2018

Pagination : 17-22

ISBN : 978-2-85420-619-7

ISSN : $1254-4590$

Référence électronique

Damien Larrouqué, «L'Administration nationale de l'éducation publique en Uruguay : une institution unique au monde », Revue internationale d'éducation de Sèvres [En ligne], 78 | septembre 2018, mis en ligne le 01 septembre 2020, consulté le 25 juin 2021. URL : http://journals.openedition.org/ries/6326 DOI : https://doi.org/10.4000/ries.6326

Ce document a été généré automatiquement le 25 juin 2021.

(C) Tous droits réservés 


\title{
L'Administration nationale de l'éducation publique en Uruguay : une institution unique au monde
}

\author{
Damien Larrouqué
}

1 De par la fonction qu'elle remplit, l'autonomie qui lui est constitutionnellement garantie, les règles qui régissent son fonctionnement ainsi que les prérogatives qui lui sont octroyées, l'Administration nationale de l'éducation publique (ANEP) en Uruguay apparait comme une institution vraisemblablement unique au monde. Fondée au sortir de la dictature (1985), l'ANEP ne se substitue pas formellement au ministère de l'éducation et de la culture $(\mathrm{MEC})$ mais phagocyte la très grande majorité de ses attributions. Organisme parapublic ayant le statut d'entité autonome, l'ANEP est responsable de la gestion des politiques éducatives uruguayennes. De la détermination des travaux d'infrastructures scolaires à l'élaboration des programmes, en passant par la fixation du salaire des fonctionnaires, l'ANEP gère, en propre, l'ensemble des questions qui touchent au monde de l'enseignement primaire comme secondaire du pays. Quant au ministère, il ne s'occupe que de la petite enfance (avant l'âge de trois ans) et de l'éducation dite «non-formelle » (programmes de formation et de réinsertion à destination des adultes). En d'autres termes, le MEC n'a d'éducation, fondamentalement, que le nom : la promotion de la culture représente le cœur de son activité. Quant à l'ANEP, sa principale particularité repose sur son caractère autogéré. Par le truchement d'élections nationales organisées sous le contrôle de la Cour électorale (Corte Electoral), le corps enseignant élit ses représentants dans les différentes instances de direction de l'ANEP. Malgré la Loi générale de l'éducation approuvée en décembre 2008 - dont l'un des objectifs était précisément de renforcer le rôle du MEC, l'immixtion politique dans les affaires éducatives reste minime en Uruguay. Pour comprendre la singularité de ce système de gestion, il convient de revenir aux fondements philosophique et politique de l'éducation uruguayenne. 


\section{Entre conceptions « varéliennes » et legs institutionnel «batlliste »}

2 Journaliste francophile influencé par le positivisme comtien, José Pedro Varela est commémoré comme le père de l'école universelle et gratuite en Uruguay (1877). Nommé directeur de l'instruction publique en mars 1876, le fondateur de la Société des amis de l'éducation populaire (1868) et auteur de L'Éducation du peuple (1874) n'a pas pu mettre en place l'intégralité de son projet éducatif. Outre la laïcité qui ne sera établie qu'une trentaine d'années plus tard, ce «Jules Ferry uruguayen » rêvait de bâtir un système scolaire décentralisé à l'extrême, où «l'action, l'initiative et la volonté des localités puissent se manifester librement pour établir, organiser, maintenir et diriger l'école publique dans tous ses aspects ». Chantre de l'autonomie politique et financière des établissements publics, José Pedro Varela a été bridé dans ses ambitions décentralisatrices par le gouvernement autoritaire de l'époque. Ses propositions éducatives n'en étaient pas moins très radicales: il considérait notamment que le pouvoir politique ne devait avoir aucun droit d'ingérence sur les questions éducatives, au risque de favoriser une politisation de l'enseignement qui nuirait à l'intérêt général. La gestion économique du système scolaire ne devait pas non plus relever de l'État, par crainte de voir les financements destinés à l'éducation s'écrouler en période de disette budgétaire. Jamais tombées dans l'oubli malgré la mort prématurée de leur auteur en 1879, ces conceptions iconoclastes n'ont eu de cesse d'être relayées par toute une génération d'enseignants et d'éducateurs uruguayens. De la fondation d'un système d'enseignement spécifique au monde rural à la création des écoles expérimentales, ces visions ont irrigué toutes les initiatives alternatives conduites au milieu du $\mathrm{XX}^{\mathrm{e}}$ siècle en Uruguay. Aujourd'hui encore, José Pedro Varela demeure une figure intellectuelle tutélaire. Ses conceptions ont forgé un cadre cognitif singulier, au prisme duquel les instituteurs ont pensé et bâti un système d'autogestion unique en son genre.

Consacrée par la Constitution, l'autonomie de l'ANEP se fonde ainsi sur le principe varélien selon lequel les affaires scolaires doivent être gérées par les principaux intéressés: les instituteurs. Toutefois, ses fondements institutionnels remontent également à l'héritage administratif laissé par José Batlle y Ordoñez, le précurseur de l'État-providence en Amérique latine. Bien qu'il n'ait gouverné que durant deux mandats (1903-1907 et 1911-1915), Batlle y Ordoñez figure en première place dans le panthéon civil uruguayen. Ayant rêvé de faire de l'Uruguay " un petit pays modèle », il a eu une si grande influence sur la vie politique, économique et sociale que l'on a pu même évoquer une batllidad uruguayenne, c'est-à-dire un vecteur d'identité et de cohésion nationales né de l'attachement au projet de société façonné par Batlle y Ordoñez et ses successeurs. Sur le plan institutionnel, le legs batlliste [batchiste] a été particulièrement important. Adepte du spiritualisme rationaliste de Karl Krause - un disciple de Kant qui a développé une vision très optimiste de l'homme, selon laquelle l'harmonie doit prévaloir dans ses rapports avec les institutions sociales et politiques (famille, Église, État), José Batlle s'est battu, sa vie durant, pour mettre sur pied un État fort, mais contrebalancé par une conception équilibrée des pouvoirs. Caractéristique du fonctionnement démocratique uruguayen, son «présidentialisme de compromis » s'est par exemple illustré, d'après le politiste Jorge Lanzaro, dans l'existence d'un exécutif collégial (1919-1933) inspiré du modèle suisse. Cinq années après la mort du leader réformateur et bien qu'adoptée dans un contexte de radicalisation autoritaire, la 
Constitution de 1934 est venue conforter l'idéal batlliste. À bien des égards, cette constitution a redessiné de manière singulière toute la géométrie institutionnelle. Depuis cette époque, l'appareil politico-administratif se décline en trois cercles de pouvoir concentriques. Le premier correspond aux institutions classiques du pouvoir démocratique (exécutif, législatif bicaméral, organes judiciaires et ministères). Le deuxième inclut les institutions décentralisées et autres entités autonomes (Entes Autónomos) directement situées dans l'orbite de l'État central. Quant au troisième cercle, il rassemble les personnes publiques non étatiques (PPNE) qui peuvent être assimilées à des agences ou à des organismes spécialisés dans des domaines bien précis tels que la recherche et le développement ou le contrôle et la régulation. Pour comprendre ce qu'est l'ANEP, il convient de ne s'attarder ici que sur ce second cercle, et plus spécifiquement sur ce statut d'entités autonomes dont elle relève.

\section{Un statut institutionnel particulier symbole du renouveau démocratique}

4 Financées par l'État, les entités autonomes sont des institutions dévolues aux services publics, mais dont la gestion jouit d'une autonomie beaucoup plus grande vis-à-vis du pouvoir exécutif que celle concédée aux administrations centrales. Dans l'esprit batlliste, la raison d'être des entités autonomes repose sur leur affranchissement vis-àvis des visions à court terme qu'institue la compétition électorale. En clair, cette reconfiguration institutionnelle vise à mettre à l'abri des logiques partisanes et autres stratégies "politico-politiciennes » certains domaines d'intervention publique considérés comme capitaux pour le développement social et économique du pays. Les entreprises publiques telles qu'Ancap (combustibles), UTE (électricité) ou Antel (télécoms), certaines banques nationalisées après la grave crise économique de $2002 \mathrm{ou}$ encore l'Université de la République (UdelaR) relèvent de ce statut.

Approuvée le 28 mars 1985, la loi n 15739 qui a engendré l'ANEP constitue l'une des toutes premières lois entérinées par la démocratie renaissante. Quelques jours auparavant, la reconnaissance des résultats présidentiels par la junte, la prise de fonction du nouveau président démocratiquement élu le $1^{\mathrm{er}}$ mars 1985 et la libération des derniers « otages » de la dictature ont marqué symboliquement le passage d'un État autoritaire à un État d'autorité, selon la belle formule de l'historien uruguayen Alvaro Rico. Au-delà de la perpétuation de l'héritage varélien, le statut d'entité autonome accordée à l'ANEP illustre le souhait du pouvoir politique de montrer sa compassion envers un monde de l'éducation qui a été particulièrement meurtri par la purge militariste (1973-1985).

Durant la dictature en effet, la part du PIB consacrée à l'éducation nationale a fondu de trois points sur l'ensemble de la période. Elle est passée de 4,1\% en 1968 à 1,4\%, seize ans plus tard. Pour prendre la mesure de cette rétorsion financière, l'involution drastique du budget alloué à chaque élève est sans doute plus significative encore : il a diminué de $30 \%$ dans le primaire, de $35 \%$ dans le secondaire, de plus de $50 \%$ dans l'enseignement technologique, et même de $70 \%$ à l'université, considérée comme le bastion de la subversion (Ifrán, 2012). Face à la précarité des salaires, les premiers "professeurs-taxis » - qui multiplient les charges d'enseignement d'une école à une autre - font leur apparition au début des années 1980. Comme tous les fonctionnaires, les enseignants sont classés en trois catégories (A, B et C), la première regroupant celle 
des individus conformes aux exigences idéologiques du régime, la seconde nécessitant un recadrage politique et professionnel de ses membres, la dernière se traduisant par une expulsion pure et simple de l'administration pour tous les infortunés qui la composent. Le plus souvent catalogués dans les deux dernières, les enseignants ont payé un lourd tribut à la junte militaire; laquelle a, du reste, pris à cœur de « renationaliser » l'Éducation. Confiée à un organe militaro-civil baptisé CONAE (Consejo nacional de Educación), la gestion éducative a fait l'objet d'une recentralisation abrupte. À partir de 1973, il a été mis un terme à l'autonomie relative et aux expériences constructivistes des décennies passées. Les méthodes d'enseignement se sont rigidifiées. Pour ce qui est des programmes, célébration de l'histoire nationale et glorification de la morale civique ont caractérisé la période.

7 Depuis sa création en 1985 et malgré quelques ajustements mineurs opérés par la Loi générale d'éducation votée en 2008, l'ANEP n'a que peu évolué dans son fonctionnement.

\section{Une structure organisationnelle hybride}

Basée sur le principe d'autogestion, l'ANEP est chapeautée par un organe exécutif baptisé Conseil directif central (Codicen). Composé de cinq professeurs reconnus pour leurs trajectoires académiques - dont trois sont directement nommés par le président de la République après validation du Parlement et deux élus par leurs pairs, le Codicen détermine et administre la politique éducative de la maternelle au lycée. À partir de son entrée en fonction et durant les cinq années d'exercice suivantes, le Codicen n'a de compte à rendre qu'au Parlement qui lui alloue les ressources. En guise d'ordre de grandeur, l'ANEP représentait 10,75\% du budget de l'État pour l'année 2005 (Vaillant, 2009 : 13). Jusqu'à l'achèvement du cycle électoral en vigueur, le pouvoir exécutif n'a donc qu'un droit de regard limité sur la politique éducative de son pays. L'unique levier d'action pour le Président passe par le bras de son ministre de l'éducation, lequel dirige la Commission coordinatrice du système national d'éducation publique (CCSNEP), créée par la Loi générale d'éducation du 12 décembre 2008. Or, rassemblant plusieurs acteurs du secteur éducatif - dont le recteur de l'UdelaR et le Président du Codicen, la CCSNEP ne fait que fixer de grandes orientations générales. Ensuite, chaque entité autonome est libre de suivre ou non les résolutions concertées au niveau ministériel.

Souverain dans les limites que lui consacre la Constitution, le Codicen peut décider de modifier les cursus, d'appliquer de nouveaux critères à la sélection des enseignants ou encore d'ajourner la réalisation de travaux d'infrastructures scolaires. Ventilant à sa guise les budgets publics, il réalise ses choix en accord avec les délégués de chaque branche d'enseignement réunis en différents "conseils». Au nombre de quatre, ces conseils d'éducation (primaire, secondaire, technologique et de formation enseignante) réunissent chacun trois professeurs - dont deux sont nommés par le Codicen et un élu par l'ensemble du corps enseignant. Il existe enfin, adossés à chaque conseil d'éducation, des organes délibératifs que l'on nomme les assemblées techniques enseignantes (ATD pour leur sigle en espagnol). Composées de plusieurs dizaines de membres élus par leurs pairs, les ATD nationales s'apparentent, selon le niveau, à des parlements d'instituteurs ou de professeurs. Dotées d'un statut consultatif, elles sont tout de même habilitées à formuler des propositions auprès de leur conseil d'éducation 
respectif. Au niveau local, des ATD existent également dans la plupart des établissements : elles défendent alors la position des enseignants face à leur direction.

Structure organisationnelle de caractère à la fois pyramidal (Codicen - conseils de l'éducation) et horizontal (conseils de l'Éducation - ATD), l'ANEP se donne à voir comme un édifice institutionnel novateur mais décrié.

\section{Un pouvoir décisionnel amoindri par la quête du consensus}

11 Certes, au niveau conceptuel, le design institutionnel de l'ANEP est très élégant : sur le papier, le principe de l'autonomie enseignante est unanimement apprécié. Toutefois, cette architecture séduisante se révèle peu fonctionnelle à l'usage: la politique éducative est ainsi difficilement gouvernable. D'après deux politistes uruguayens, "l'autonomie des conseils d'enseignement est un obstacle à la réalisation des transformations éducatives qui s'imposent » (Bentancourt et Mancebo, 2011). En effet, à travers ses multiples instances de consultation, l'ANEP semble reposer sur plusieurs pierres d'achoppement qui constituent autant de points de veto. Pour reprendre une expression du célèbre politiste nord-américain Georges Tsebelis, l'ANEP ressemble à " constellation d'acteurs-veto", dans le sens où l'accord de chacun d'entre eux est nécessaire pour faire évoluer le statu quo. Dès lors que toutes les décisions se prennent par consensus et que l'ensemble des acteurs concernés font montre d'un attachement important à leur autonomie respective, la résistance au changement apparaît plus forte encore au sein de l'ANEP qu'elle ne l'est dans les administrations bureaucraticomonocratiques classiques - ces dernières étant, d'après Max Weber, "la forme de domination la plus rationnelle du point de vue formel ", mais s'avèrent, dans la pratique, minées par leur manque de souplesse institutionnelle.

12 Très concrètement, avant d'être approuvée par le Codicen en concertation avec le conseil d'enseignement concerné, toute initiative éducative d'envergure doit avoir reçu l'aval de l'ATD correspondante, laquelle ne se réunit en moyenne qu'une fois par an. De surcroît, les suffrages que ses délégués ont reçus de l'ensemble du corps professoral confèrent aux ATD, malgré leur statut consultatif, une très grande légitimité que les membres du Codicen, professeurs eux-mêmes, ne sauraient offenser au risque de se renier. En résumé, l'idéal de participation démocratique (la valorisation du dialogue), le perfectionnisme intellectuel (l'excessive méticulosité) et la connivence voire complaisance académique (le respect envers l'opinion des collègues) ont plongé la politique éducative uruguayenne dans une profonde inertie depuis plus de vingt ans. En somme, l'ANEP manquerait cruellement de capacité décisionnelle.

$13 \mathrm{Au}$ demeurant, si les difficultés à réformer l'éducation uruguayenne jouent contre la volonté des politiques d'améliorer un système d'enseignement souvent présenté comme déliquescent, l'ingouvernabilité chronique de l'ANEP lui a permis de se prémunir contre les velléités de néo-libéralisation qui ont affecté les autres systèmes éducatifs de la région au cours des années 1990 et 2000. 


\section{Pour le pire et pour le meilleur, un système résistant au changement} politique et sociale, il est indéniable que sa qualité s'est dégradée au cours des vingt dernières années, notamment au niveau secondaire (Filardo, 2014). La littérature spécialisée fait état d'une augmentation préoccupante de la "désertion scolaire »; c'est-à-dire de l'abandon prématuré de la scolarité avant l'âge légal. On note également une tendance, certes beaucoup moins marquée qu'ailleurs en Amérique latine, de migration des élèves des établissements publics vers les instituts d'enseignement privés. En 2008, selon les données du ministère, les écoles primaires uruguayennes sous contrat accueillaient 15,7 \% du contingent scolaire contre 12,6 \% en 2001. De surcroît, entre 2008 et 2009, l'éducation privée a vu ses effectifs augmenter de 2,2\% tandis que l'éducation publique a symétriquement perdu $2 \%$ des siens. surannée. Elle est ainsi considérée comme "endogame, autoréférencé et encapsulé " (Marrero, 2008); dans le sens où les professeurs chargés d'inculquer les rudiments du métier à leurs futurs collègues font très peu appel à un renouvellement des méthodes pédagogiques et se montrent très hermétiques à toute volonté d'innovation. En outre, l'avancement de carrière se base uniquement sur la logique de l'ancienneté, et en aucune façon sur les compétences acquises ou l'esprit d'initiative. Plus inquiétant encore, depuis que le pays est sorti, au milieu des années 2000, du marasme économique, la profession susciterait de moins en moins de vocations.

résumé, affranchi des contingences politico-électorales comme indépendant des gouvernements qui en sont le produit, le système éducatif uruguayen, autogéré par l'ANEP, est peu sensible aux injonctions de transformation et autres exigences d'adaptation. Or, cette très forte résistance au changement n'est pas que problématique : elle a aussi du bon. En l'espèce, la pénétration des idées néolibérales a été beaucoup moins profonde en Uruguay que dans d'autres pays d'Amérique latine. Du moins, elle ne s'est pas traduite par un processus de décentralisation éducative (Mancebo, 1998), mais par une réforme hétérodoxe plus consensuelle qu'ailleurs (Lanzaro, 2006). Même cent cinquante après la mort de son fondateur, l'école uruguayenne ne s'est encore jamais départie de ses principes varéliens : elle demeure républicaine, laïque, gratuite, obligatoire et ouverte à tous.

n forme d'ouverture, on pourrait même considérer le principe de l'autogestion éducative à l'uruguayenne comme un possible modèle à étendre. Alors que la gouvernance éducative reste encore dominée, à l'échelle internationale, par « l'agenda néolibéral " associé aux logiques de dérégulation, décentralisation, privatisation et autres market-oriented reforms (Ozga et Segerholm, 2015), il apparaît sans doute préférable d'en confier la conduite, le contrôle et l'évaluation à des assemblées de professeurs plutôt qu'à des agences déconnectées du monde éducatif ou à des cabinets de conseil privés. 


\section{BIBLIOGRAPHIE}

BENTANCUR N., MANCEBO M. E. (2011) : « La educación en la encrucijada : escrutinio político y gobierno de la enseñanza », in CAETANO G. et al. Políticas en tiempo de Mujica : en busca del rumbo, Montevideo : Estuario Editora, 2011, p. 124-130.

FILARDO V. (2014) : «L'éducation secondaire, le talon d'Achille de l'Uruguay », Cahiers des Amériques latines, $\mathrm{n}^{\circ} 77$, p. 49-68.

IFRÁN D. (2012) : De Varela al Plan Ceibal : Genealogía, reflexiones y vivencias de la enseñanza pública uruguaya, Montevideo : Piscolibros.

LANZARO J. (2006) : « La reforma educativa en Uruguay (1995-2000) : virtudes y problemas de una iniciativa heterodoxa ", in FRANCO R., LANZARO J. (dir.), Política y políticas públicas en los procesos de reforma de América Latina, Buenos Aires : Miño y Dávila Editores, p. 247-286.

MANCEBO M. E. (1998) : « Las políticas educativas de Uruguay en el contexto latino-americano (1985-1994) », Revista Uruguaya de Ciencia Política, nº 10, p. 101-115.

MARRERO A. (2008) : « La herencia de nuestro pasado : reflexiones sobre la educación del siglo XX », in NAHUM B. (dir.), El Uruguay del siglo XX. vol. III : La sociedad, Montevideo : Ediciones de la Banda Oriental, p. 45-75.

OZGA J. et SEGERHOLM C. (2015) : « Neo-liberal agenda(s) in education » in GREK S., LINDGREN J. (dir.), Governing by Inspection, New York : Routledge, p. 27-37.

VAILLANT D. (2009) : Los sindicatos y la educación publica en Uruguay, Rio de Janeiro : Fundação Konrad Adenauer.

\section{INDEX}

Mots-clés : politique éducative, système éducatif, autogestion

Palabras claves : política educacional, sistema educativo, autogestión

Keywords : educational policy, educational system, self government Index géographique : Uruguay

\section{AUTEUR}

\section{DAMIEN LARROUQUÉ}

Damien Larrouqué est attaché temporaire d'enseignement et de recherche (ATER) à l'Université Paris 2 Panthéon-Assas, docteur associé au Centre de recherches internationales (CERI-Sciences Po) et membre du bureau exécutif de l'Observatoire politique de l'Amérique latine et des Caraïbes (OPALC-Sciences Po). Ses recherches portent sur les transformations de l'État et de l'action publique en Amérique latine, ainsi que sur l'évolution des partis politiques, notamment en Argentine, au Paraguay et en Uruguay. Courriel : damien.larrouque@sciencespo.fr 